\title{
A novel model of periventricular leukomalacia on mouse organotypic brain slice culture
}

\author{
Tsupykov O. M. ${ }^{1,2}$, Lushnikova I. V. ${ }^{1}$, Nikandrova Y. A. ${ }^{1}$, Yatsenko K. V. ${ }^{1}$, Ustymenko A. M. ${ }^{2}$, Kyryk V. M. ${ }^{2}$, \\ Butenko G. M. ${ }^{2}$, Skybo G. G. ${ }^{1,2}$ \\ ${ }^{1}$ Bogomolets Institute of Physiology NAS of Ukraine, Kyiv, Ukraine \\ ${ }^{2}$ State Institute of Genetic and Regenerative Medicine NAMS of Ukraine, Kyiv, Ukraine \\ e-mail: tsupykov@gmail.com
}

\section{ABSTRACT}

The creation of adequate in vitro and in vivo models of neural tissue injury is essential to assess the therapeutic effect of pharmacological agents and regenerative potential of various types of stem cells in diseases of the central nervous system. The aim of this work was to create a novel model of cerebral white matter lesions - periventricular leukomalacia (PVL) - on murine organotypic brain slice culture.

MATERIALS AND METHODS. The PVL model was developed on cultured organotypic mice brain slices subjected to oxygen-glucose deprivation (OGD) followed by addition of endotoxin lipopolysaccharide (LPS) in the culture medium. To analyze the degree of tissue injury within PVL simulation, we used spectrophotometric method for estimation of cytosolic enzyme lactate dehydrogenase (LDH) in the culture medium and immunohistochemical analysis of the slices using antibodies to Rip, GFAP and Iba-1 protein markers of oligodendrocyte, astroglia and microglia, respectively.

RESULTS. It was shown that the combined effect of OGD and lipopolysaccharide resulted in a significant release of the cytosolic enzyme LDH in culture medium, decrease of Rip-immunoreactivity and a pronounced reactive astro-and microgliosis in murine organotypic brain slice culture.

CONCLUSIONS. Our model of PVL developed on cultured organotypic mice brain slices is novel and promising tool to study pathogenic mechanisms of cerebral white matter lesions and ways of neuroprotection in this pathology, including pharmacological agents and transplantation of stem cells.

KEYWORDS: periventricular leukomalacia; lipopolysaccharide; oxygen-glucose deprivation; organotypic brain slice culture

One of the most actual health and social problems of modern neurology and pediatrics are perinatal central nervous system (CNS) lesions, including injury of the cerebral white matter in premature newborns periventricular leukomalacia (PVL). PVL leads to a variety of neurological disorders, including motor and cognitive disorders, and nearly $90 \%$ of cases cause cerebral palsy [1]. To study the pathogenic mechanisms of periventricular leukomalacia and ways of brain neuroprotection, a variety of in vivo and in vitro experimental models were created $[7,8,11,18,20]$

A hypoxic-ischemic model of brain white matter lesion in newborn rodents is most often used to simulate PVL in vivo [3, 14]. It was shown that the most sensitive cells that are damaged by PVL are oligodendrocytes. Therefore, the dissociated culture of oligodendrocyte or oligodendrocyte progenitors treated by neuroinflammation inductor endotoxin lipopolysaccharide (LPS) is the most common PVL in vitro model used up to now $[12,13,16]$.
In addition to dissociated cells culture, acute tissue slices or organotypic cultures are commonly used for neurobiological studies in vitro. Each of these in vitro models has its advantages and disadvantages. The first studies on acute brain slices that were incubated in saline were carried out in 1924 [25]. It was shown that such slices in vitro retain their viability, and their metabolic and electrophysiological characteristics coincide with similar characteristics of intact brain tissue [9]. However, acute slices quickly lose their viability, therefore have very limited time for research.

The advantage of dissociated cell culture is the possibility of providing «pure» culture, which contains a specific cell type, and monitoring changes in morphological and electrophysiological characteristics in a separate cell. Such properties of dissociated cultures are especially valuable for studying the mechanisms of certain processes. The disadvantages of such a culture are that the cells are no longer an integral part of the primary neuronal network; and 
their morphology, metabolism and reactions to certain influences change [19].

Most suitable for modeling of pathological processes in vitro and developing strategies of their correction are organotypic cultures $[5,26]$. Organotypic brain slice culture is increasingly becoming an alternative to experiments in vivo. Organotypic brain culture remains cytoarchitectonics, types of cells and layers, primary intercellular connections, synaptic organization, location of receptors etc. [15]. The advantages of such a culture also include the possibility of a local electrical or chemical stimulation of certain parts of neurons (body, axons, dendrites); fine surgical manipulation with a slice (isolation of individual parts, dissecting of afferent paths, separation of processes from cell's body). In addition, the present organotypic culture provides a direct access to the extracellular space, which allows to control easily conditions of the tissue, and provides a direct impacts on tissue by various substances of desired concentration [10].

The advantages of organotypic culture appear attractive for the modeling of different pathological states of the CNS, particularly PVL. The use of hippocampal organotypic culture for in vitro modeling of PVL has been reported earlier [8]. However, it is well known that PVL affects mostly myelinated nerve fibers of the corpus callosum, which is missing in hippocampal organotypic culture [6]. Therefore, we decided to develop in vitro model of PVL utilizing organotypic culture of brain slices, where the corpus callosum remains preserved.

Recently, increasing attention is paid toward the research of the possibility to utilize stem cells in the complex treatment and rehabilitation of patients with CNS diseases. It is thought that the dysfunction of resident progenitors plays a significant role in the pathogenesis of perinatal brain lesions, while activation or substitution of these cells may promise a positive therapeutic effect $[2,4,21,23,24]$. To explore the response of nervous tissue on the transplantation of stem cells deriving from various sources, organotypic culture of brain slices can be useful tool when modeling many CNS disorders including PVL. This experimental system can be useful during the research of contact and non-contact (humoral) interaction of transplanted stem cells with recipient tissue.

Therefore, the aim of the study was to develop a model of periventricular leukomalacia in murine organotypic brain slice culture.

\section{MATERIALS AND METHODS}

All experiments were performed in accordance with international principles of the European Convention for the Protection of vertebrate animals used for experimental and other scientific purposes (European convention, Strasburg, 1986), Article 26 of the Law of Ukraine «On protection of animals from cruelty» (21.02.2006) and all norms of bioethics and biosafety. The FVB «wild» type mice are kept under standard conditions with free access to food and water ad libitum at the experimental clinic of State Institute of Genetic and Regenerative Medicine NAMS.

Organotypic brain slice culture of newborn mice. Organotypic brain slice culture was obtained from FVB mice of postnatal day 7. After euthanasia of animals, the brain was isolated by rapid decapitation, divided into two parts on the median line and cut into 350 microns thick slices with automatic chopper (Mclllwain, GB). The sections were cultured in 6-well plates on porous Millicell CM (Millipore, USA) culture plate inserts in a $\mathrm{CO}_{2}$ incubator at $35{ }^{\circ} \mathrm{C}$ on the gas-liquid interface: a mixture of air with $5 \% \mathrm{CO}_{2}$ versus liquid medium containing $50 \% \mathrm{MEM}$, $25 \%$ Hanks balanced salt solution, $25 \%$ inactivated horse serum, 10 mM Tris, 2 mM NaHCO3, 12.5 mM HEPES, 15 mM glucose, $100 \mathrm{U} / \mathrm{ml}$ penicillin, $100 \mu \mathrm{g} / \mathrm{mL}$ streptomycin (all - Sigma-Aldrich, USA) at pH 7.2. The culture medium was changed on the second day of incubation and then twice a week.

Modelling of periventricular leukomalacia on organotypic brain slice culture of FVB mice. Periventricular leukomalacia was modeled by oxygen-glucose deprivation (OGD) of the brain slices, followed by addition of endotoxin lipopolysaccharide into culture medium to simulate the process of inflammation. For the OGD, slices on inserts were placed into special chamber where the gas atmosphere contained $95 \%$ nitrogen and $5 \% \mathrm{CO}$, while liquid medium contained PBS, $12.5 \mathrm{mM}$ Hepes and $15 \mathrm{mM}$ D-sucrose substituting glucose. After $30 \mathrm{~min}$ of OGD slices were washed twice and returned to normal culture conditions (normoxic reoxygenation for 24 and 48 hours). Lipopolysaccharide (LPS) (L4130, Sigma-Aldrich, USA) was added into the culture medium after OGD. In order to determine the impact of different LPS concentrations on the state of organotypic brain slices, three different concentration (10, 100 and $1000 \mathrm{ng} / \mathrm{ml}$ ) was studied in pilot experiments.

Quantitative analysis of cytosolic lactate dehydrogenase (LDH) in the culture medium. To determine the changes in the relative amount of LDH in culture medium in response to the treatment with various concentrations of LPS in the combination with or without OGD, $200 \mu \mathrm{l}$ of culture medium was collected into 24-well plate at 24 and 48 hours after OGD. Samples were collected in duplicates. CytoTox Non-Radioactive Cytotoxicity Assay kit (Promega, USA) was utilized for the colorimetric method that was performed as follows: the reaction was initiated by adding $200 \mu$ of substrate in each well and incubated at room temperature in the darkness for 30 minutes; the reaction was terminated by addition of $200 \mu \mathrm{l}$ Stop Solution.

Optical density of the samples was measured with a spectrophotometer uniSPEC 2 ( $L L G$, Germany) at wavelength of $492 \mathrm{~nm}$. We determined the average values of duplicates for each well. As controls we used: 1 - culture medium from the wells without organotypic culture (optical density value of which was subtracted from that of obtained from experimental wells); 2 - culture medium from the well with untreated LPS and OGD cultures. Changes in the relative LDH amount in culture medium was expressed in arbitrary units that represent units of solution optical density normalized to the tissue area in the respective well.

Immunohistochemical staining of organotypic brain slice cultures. Assessment of various glial cells types in cultured brain slices under PVL model was conducted by immunohistochemistry. Organotypic murine brain slice cultures were fixed with $4 \%$ solution of paraformaldehyde in $0.1 \mathrm{M}$ PBS. Fixed slices were kept in blocking non-specific binding of proteins solution containing $0.1 \mathrm{M} \mathrm{PBS}(\mathrm{pH} 7.4), 0.5 \%$ bovine serum albumin (BSA) and $0.3 \%$ Triton X-100 (Sigma-Aldrich, USA). The incubation of slices in the primary antibodies solution lasted for 12 hours at $+4{ }^{\circ} \mathrm{C}$. We used the following primary antibodies: anti-GFAP (a marker of astrocytes) 1: 1500 (Dako Cytomation, Denmark), anti-Iba-1 (marker of microglia) 1: 1000 (Wako, Japan), Anti-Rip (marker of oligodendrocytes) 1: 200 (Abcam, USA). Relevant secondary antibodies, conjugated with fluorochrome AlexaFluor (Invitrogen, USA), visualized primary antibodies. Stained organotypic brain slice culture was embedded in a drop of Immu-Mount ${ }^{\mathrm{TM}}$ mounting medium (Thermo Scientific, USA). Immunohistochemically stained culture was studied using confocal scanning microscope FV1000-BX61WI (Olympus, Japan).

Quantitative image analysis was carried out using the software ImageJ (National Institutes of Health, USA). The intensity and area of fluorescence marker was measured by automatic calculation of the average value of gray within the measurement threshold. The results were presented as integrated density of fluorescence in arbitrary units, which are equal to the fluorescence intensity multiplied by area of fluorescence (excluding the integrated density of the background fluorescence).

Statistical analysis. Statistical analysis was performed using the Origin Pro 8.5 software (OriginLab Corp., USA). Data sampling included the results obtained from 3 experiments. The results are shown as mean from four values $(n=4)$ in each experimental group \pm standard error of the mean (SEM). The data were characterized by normal distribution, the statistical probability of differences was determined by paired Student t-test; the differences were considered significant at $p<0.05$. 

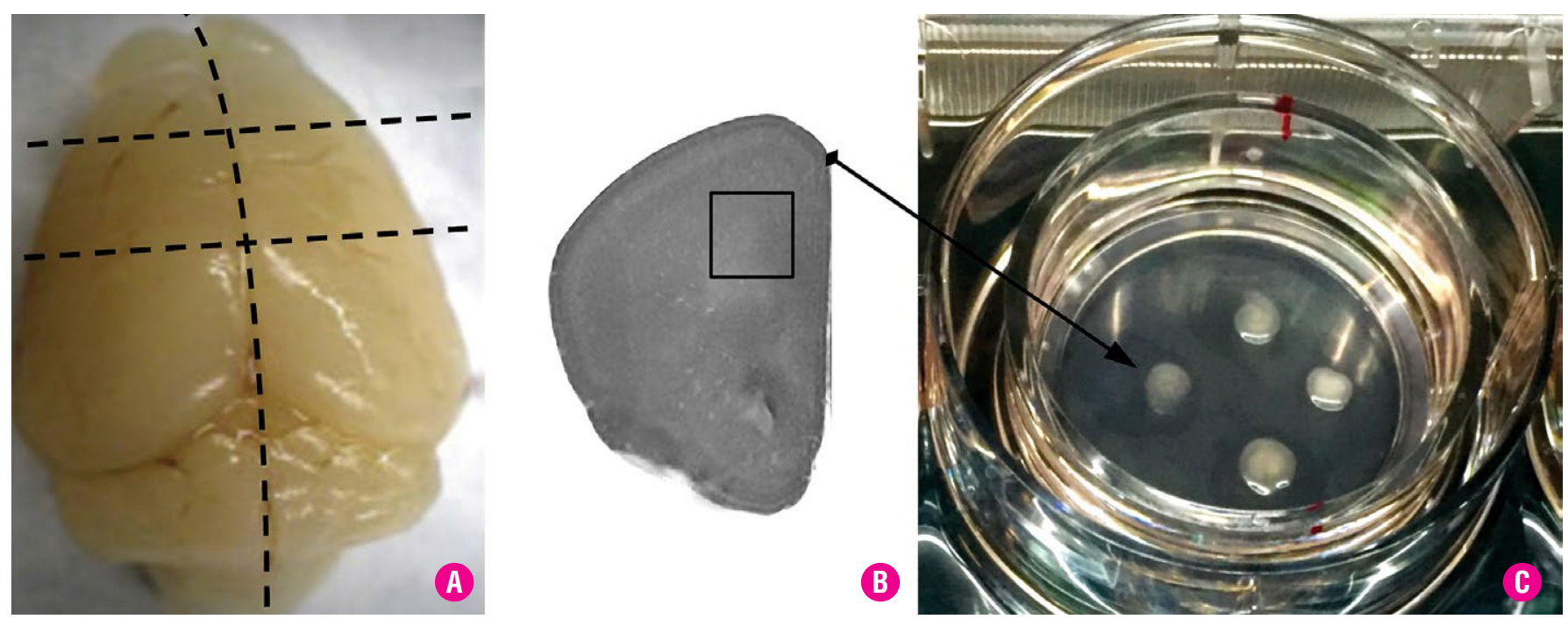

入

Fig. 1. Photo of organotypic murine brain slice cultures. A - the mouse brain. Intermittent line shows the areas from which front slices for organotypic brain cultures were made. B - a half of the front cut. Squares mark an area that was taken for immunohistochemical analysis. C - cultured brain slices on a membrane of culture inserts

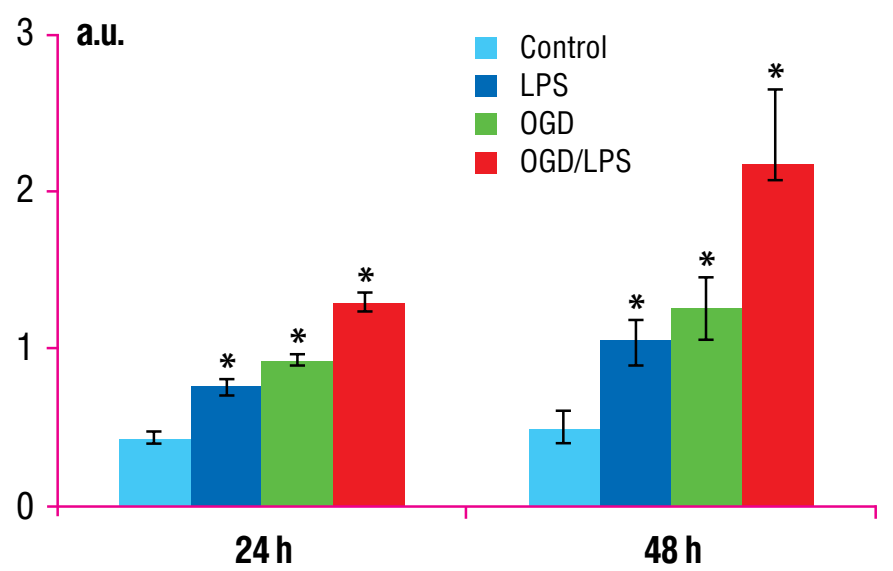

Fig. 2. The relative amount of LDH enzyme in the culture medium of organotypic slices 24 and 48 hours after OGD, LPS addition or combined procedure of OGD and LPS.

Note: ${ }^{*}$ - statistically significant difference compared with control $(p<0.05)$

\section{RESULTS AND DISCUSSION}

After isolation, we divided brain into two parts along the midline and cut into front slices $(350 \mu \mathrm{m})$ in the anteroposterior direction from bregma 1.10 to bregma 0.10 (Fig. 1-A).

Slices obtained from indicated zone of each brain hemisphere (Fig. 1-B), were transferred onto inserts with porous semitransparent membranes for cultivation (Fig. 1-C). It is known that periventricular leukomalacia mostly damages myelinated nerve fibers of the corpus callosum, which connects the cerebral hemispheres [6]. Therefore, we performed immunohistochemical analysis in the zone of lateral ventricles with corpus callosum (Fig. 1-B, marked with square).

\section{VIABILITY ASSESSIMENT BY LDH}

In our previous work [22] we showed that for the modelling of PVL in vivo, neuroinflammation is required in addition to the hypoxic-ischemic injury of neural tissue. Therefore, at modelling of PVL in vitro, in addition to OGD, we also added endotoxin LPS to create conditions that simulate neuroinflammation.
To determine the minimal effective concentration of LPS affecting organotypic brain slices culture, we conducted pilot experiments with three LPS concentrations $(10,100,1000 \mathrm{ng} / \mathrm{mL})$. The LPS dose $10 \mathrm{ng} / \mathrm{mL}$ did not show detectable effect on the relative amount of LDH in the culture medium compared to control. In contrast, doses of 100 and $1000 \mathrm{ng} / \mathrm{mL}$ affected the viability of cultures to similar degree (data are not shown). Thus, LPS concentration $100 \mathrm{ng} / \mathrm{mL}$ was chosen for further experiments. Organotypic slices were assigned into few groups following options of treatment: OGD, LPS $(100 \mathrm{ng} / \mathrm{mL})$ and the combined procedure of OGD with LPS

Analysis of $\mathrm{LDH}$ relative level in the culture medium was performed by colorimetric method at 24 and 48 hours after treatment, when the effects on viability were quite pronounced. During the injury of cell membrane, cytosolic enzyme LDH releases in the culture medium and indicating the degree of cell damage. The color intensity is directly proportional to the amount of the LDH in the culture medium and inversely proportional to cell viability in the culture.

Spectrophotometric analysis showed that 24 hours after adding of LPS in the culture medium the relative LDH number increased to $0.76 \pm$ 0.05 a.u. comparing to the control $-0.430 \pm 0.04$ a.u. (Fig. 2).

OGD had greater damaging effect on the tissue of culture slices and the relative amount of LDH in the culture medium reached $0.929 \pm 0.03$ a.u. Combined action of OGD and LPS led to greater raise of the relative $\mathrm{LDH}$ amount that reached 1,299 $\pm 0,07$ a.u. in 24 hours.

In 48 hours, LDH level increased significantly in all groups reaching $1.050 \pm 0.14$ a.u. after LPS treatment alone, $1.253 \pm 0.20$ a.u. after $0 G D$ alone, $2.164 \pm 0.49$ a.u. after combined treatment with both.

Thus, the results of spectroscopic analysis revealed that combined procedure of treatment with both OGD and LPS had significantly greater impact on organotypic slices than separate applications. The effects appeared after 24 hours and amplified after 48 hours.

\section{EVALUATION OF RIP EXPRESSION}

The main feature of periventricular leukomalacia is a destruction of brain white matter near the lateral ventricles. Therefore, the next step in our study was the immunohistochemical analysis of oligodendrocytes in organotypic brain slices culture.

Immunohistochemical analysis was performed 48 hours after treatments, when the impact on the viability (by the LDH levels) was the most pronounced. It was found that the addition of endotoxin LPS into the culture medium reduced Rip-immunoreactivity of the brain slices compared to control (5.92 \pm 1.05 a.u. vs. $2.67 \pm 0.28$ a.u., respectively) (Fig. 3). 

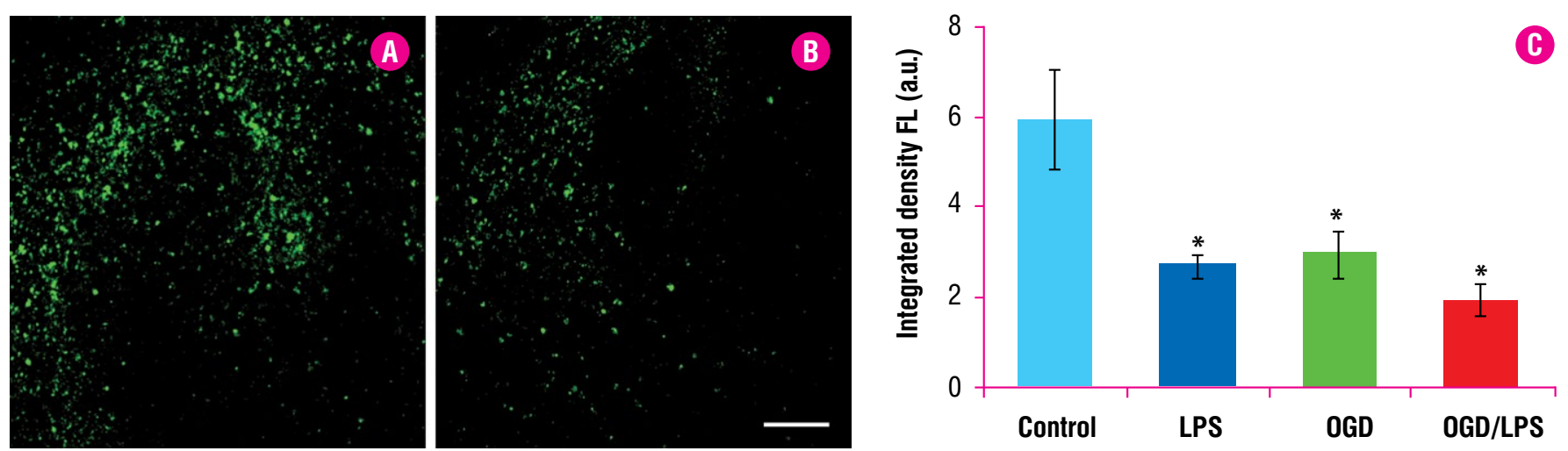

A Fig. 3. Immunohistochemical analysis of oligodendrocytes in organotypic slice culture of mouse brain. Confocal images of slices in control (A) and 48 hours after OGD/LPS treatment (B); scale - $500 \mu \mathrm{m}$. Immunostaining for oligodendrocytes marker Rip (green). C - histogram of integrated fluorescence density. Note: * - Statistically significant difference compared with control $(p<0.05)$.
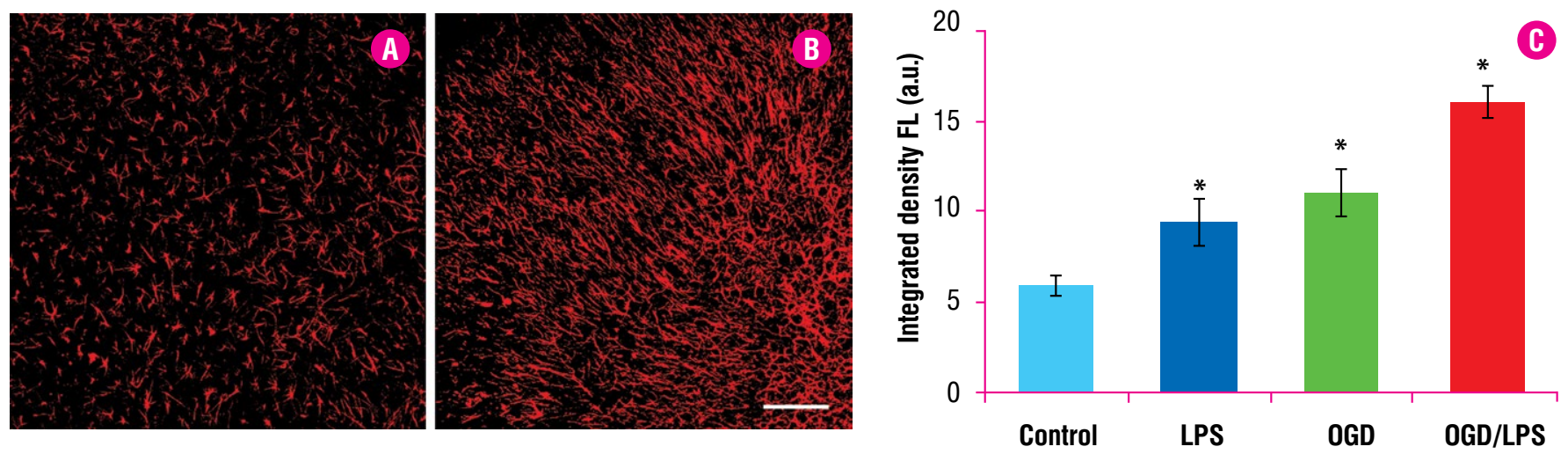

A Fig. 4. Immunohistochemical analysis of astrocytes in organotypic slice culture of the mouse brain. Confocal images of slices in control (A) and 48 hours after OGD/LPS treatment (B); scale - $500 \mu \mathrm{m}$. Immunostaining for astrocytic marker GFAP (red). C - histogram of integrated fluorescence density. Note: ${ }^{*}$ - Statistically significant difference compared with control $(p<0.05)$.
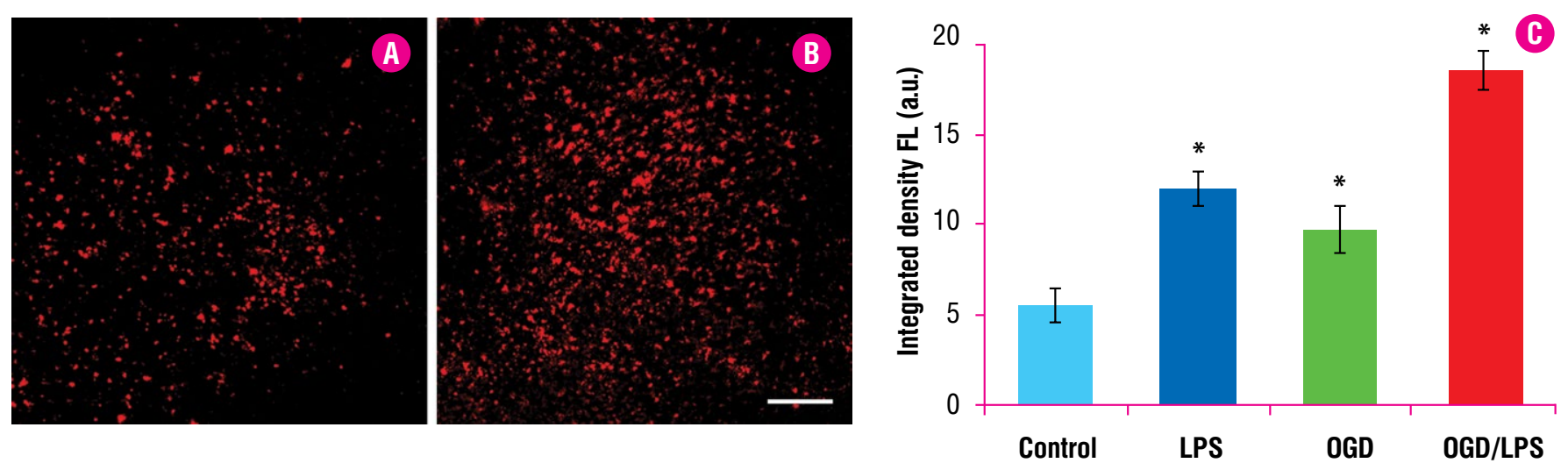

A Fig. 5. Immunohistochemical analysis of microglial cells in organotypic slice culture of the mouse brain. Confocal images of slices in control (A) and 48 hours after OGD/LPS impact (B); scale - $500 \mu \mathrm{m}$. Immunostaining for astrocytes marker Iba-1 (red). C - histogram of integrated fluorescence density. Note: * - Statistically significant difference compared with control $(p<0.05)$.

Also, OGD had a negative impact on the nervous tissue, as indicated lower level of integrated density of Rip-immunofluorescence $(2.93 \pm$ 0.53 a.u.)

Rip-immunoreactivity of brain slices was the lowest in the conditions of combined OGD/LPS action (1.90 $\pm 0.35 \mathrm{a}$. u) pointing at the significant damage of cerebral white matter during PVL modeling.

\section{EVALUATION OF REACTIVE GLIOSIS IN MURINE}

ORGANOTYPIC BRAIN SLICE CULTURE

Reactive gliosis is a nonspecific reactive change of glial cells (astrocytes and microglia) in response to the nervous tissue damage that is usually characterized by proliferation and/or hypertrophy of the glial cells [17]. In periventricular leukomalacia model in vivo, we have shown that it is accompanied by a pronounced reactive gliosis [22]. Therefore, to assess reactive gliosis in PVL model in vitro, we performed immunohistochemical study of the brain slices using antibodies to markers of astrocytes (GFAP) and microglial cells (Iba-1).

Immunohistochemical analysis of organotypic brain slice cultures revealed treatment with LPS or OGD resulted in an increase of the integrated density of GFAP-positive signal comparing to control group $(9.33 \pm 1.25,10.86 \pm 1.20$ a.u. and $5.82 \pm 0.65$ a.u., respectively) (Fig.4). 
Combined action of OGD and LPS triggered even greater reactive astrogliosis resulting in the increase of integrated fluorescence density up to $15.81 \pm 0.74$ a.u. (Fig. 4). Such an increase in GFAP-positive signal can be attributed to the hypertrophy of astrocytic soma and processes, as well as to astrocytes proliferation.

Immunohistochemical analysis of brain slices for the expression of microglia marker Iba-1 that separate treatment with LPS and OGD resulted in the elevation of Iba-1 immunopositive signal comparing to control, and corresponded to following levels of integrated fluorescence density: $11.93 \pm$ $0.91,9.74 \pm 1.28$ a.u. and $5.53 \pm 0.93$ a.u., respectively (Fig. 5).

The protocol of combined treatment with OGD and LPS promoted further reactive microgliosis with integrated fluorescence density of lba-1 positive signal reaching $18.59 \pm 1.07$ a.u. (Fig. 5).

\section{CONCLUSIONS}

A novel and promising model of periventricular leukomalacia in vitro was developed utilizing murine organotypic brain slice culture. It was shown that the combined effect from lipopolysaccharide endotoxin treatment and oxygen-glucose deprivation had the most damaging effect on the nervous tissue comparing to the separate treatment with each of these factors. Combined treatment with OGD and LPS resulted in a significant release of the cytosolic enzyme LDH into the culture medium, a pronounced reactive gliosis and decrease of Rip-immunoreactivity indicating the damage of the white matter and neuroinflammation of the brain.

The created in vitro PVL model can be used in the study of pathogenetic mechanisms of periventricular leukomalacia and ways of cerebral neuroprotection in this pathology, including promising pharmacological agents and transplantation of stem cells.

\section{REFERENCES}

1. Blumenthal I. Periventricular leukomalacia: a review. Eur J Pediatr. 2004; 163(8):435-42.

2. Cefalo MG, Carai A, Miele E, et al. Human iPSC for Therapeutic Approaches to the Nervous System: Present and Future Applications. Stem Cells Int. 2016; $2016: 4869071$.

3. Cheng T, Xue X, Fu J. Effect of OLIG1 on the development of oligodendrocytes and myelination in a neonatal rat PVL model induced by hypoxia-ischemia. Mol Med Rep. 2015;11(4):2379-86.

4. Chohan MO, Moore H. Interneuron Progenitor Transplantation to treat CNS dysfunction. Front Neural Circuits. 2016; 10:64.

5. Daviaud N, Garbayo E, Lautram N, et al. Modeling nigrostriatal degeneration in organotypic cultures, a new ex vivo model of parkinson's disease. Neuroscience. 2014; 256:10-22.

6. $\quad$ Deng W, Pleasure J, Pleasure D. Progress in periventricular leukomalacia. Arch Neurol. 2008; 65(10):1291-95.

7. Fogal B, McClaskey C, Yan S, et al. Diazoxide promotes oligodendrocyte precursor cell proliferation and myelination. PLoS One. 2010; 5(5):e10906.

8. Fu X, Zunich SM, O'Connor JC, et al. Central administration of lipopolysaccharide induces depressive-like behavior in vivo and activates brain indoleamine 2,3 dioxygenase in murine organotypic hippocampal slice cultures. J Neuroinflammation. 2010; 7:43.

9. Hertz L. Metabolic studies in brain slices - past, present, and future. Front Pharmacol. 2012; 3:26.

10. Humpel C. Organotypic brain slice cultures: a review. Neuroscience. 2015; 305: 86-98.

11. Kumral A, Iscan B, Engur D, et al. Intranasal surfactant protein $D$ as neuroprotective rescue in a neonatal rat model of periventricular leukomalacia. $J$ Matern Fetal Neonatal Med. 2016 May 16:1-6. [Epub ahead of print].

12. Lehnardt S, Lachance C, Patrizi S, et al. The toll-like receptor TLR4 is necessary for lipopolysaccharide-induced oligodendrocyte injury in the CNS. J Neurosci. 2002; 22(7):2478-86.

13. Li J, Ramenaden ER, Peng J, et al. Tumor necrosis factor alpha mediates lipopolysaccharide-induced microglial toxicity to developing oligodendrocytes when astrocytes are present. J Neurosci. 2008; 28(20):5321-30.

14. Liu $X B$, Shen $Y$, Pleasure $D E$, et al. The vulnerability of thalamocortical circuitry to hypoxic-ischemic injury in a mouse model of periventricular leukomalacia. BMC Neurosci. 2016; 17:2.

15. Morin-Brureau MM, De Bock F, Lerner-Natoli M. Organotypic brain slices: a model to study the neurovascular unit micro-environment in epilepsies. Fluids Barriers CNS. 2013; 10:11.

16. Pang $Y$, Cai Z, Rhodes $P G$. Effects of lipopolysaccharide on oligodendrocyte progenitor cells are mediated by astrocytes and microglia. J Neurosci Res. 2000; 62(4):510-20.

17. Pivneva TA, Tsupykov OM, Pilipenko MN, et al. Structural modifications of astrocytes in the hippocampus after experimental cerebral ischemia in gerbils. Neirofiziologiya. 2005; 37(5/6):410-415.

18. Rumajogee P, Bregman T, Miller SP, et al. Rodent Hypoxia-Ischemia Models for Cerebral Palsy Research: A Systematic Review. Front Neurol. $2016 ; 7: 57$.

19. Seibenhener ML, Wooten MW. Isolation and culture of hippocampal neurons from prenatal mice. J Vis Exp. 2012; 65:3634.

20. Steck J, BluemI C, Kampmann S, et al. Retinal vessel pathologies in a rat model of periventricular leukomalacia: a new model for retinopathy of prematurity? Invest Ophthalmol Vis Sci. 2015; 56(3):1830-41.

21. Syed FI, Couriel DR, Frame D, et al. Central Nervous System Complications of Hematopoietic Stem Cell Transplant. Hematol Oncol Clin North Am. 2016; 30(4):887-98.

22. Tsupykov OM, Kyryk VM, Ustymenko AM, et al. Effect of transplantation of adipose-derived multipotent mesenchymal stromal cells on the nervous tissue and behavioral responses in a mouse model of periventricular leukomalacia. Cell and organ transplantology. 2015; 3(1):68-73.

23. Tsupykov OM, Kyryk VM, Mamchur AA, et al. Transplantation of neural progenitor cells stimulates endogenous neurogenesis in mice after ischemic stroke. Cell and organ transplantology. 2014; 2(1):85-89.

24. Vadori M, Denaro L, D'Avella D, et al. Indications and prospects of neural transplantation for chronic neurological diseases. Curr Opin Organ Transplant. 2016: 21(5):490-6.

25. Warburg 0, Posener K, Negelein E. Ueber den Stoffwechsel der tumoren. Biochem Z. 1924; 152:319-344.

26. Wilhelmi E, Schöder UH, Benabdallah A, et al. Organotypic brain slice cultures from adult rats: approaches for a prolonged culture time. Altern Lab Anim. 2002; 30(3):275-83. 


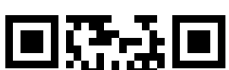

arTILLE ON THE SITE

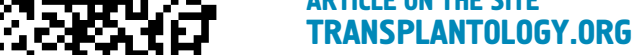

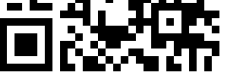

The authors indicate no potential conflicts of interest.

Received: October 03, 2016

Accepted: November 10, 2016 